\title{
Fluorodeoxyglucose Positron Emission Tomography in Primary Thyroid Lymphoma with Coexisting Lymphocytic Thyroiditis
}

\author{
Mohammad Arabi, ${ }^{1,2}$ Ryan Dvorak, ${ }^{1,2}$ Lauren B. Smith, ${ }^{3}$ Lisa Kujawski, ${ }^{4}$ and Milton D. Gross ${ }^{1,2}$
}

Background: Primary thyroid lymphoma is an uncommon neoplasm frequently associated with lymphocytic thyroiditis (LT). Once the pathologic diagnosis of primary thyroid lymphoma is established, imaging plays an important role in tumor staging and evaluating treatment response. The present case discusses the role of fluorodeoxyglucose positron emission tomography $\left({ }^{18} \mathrm{~F}-\mathrm{FDG}\right.$ PET)/computed tomography (CT) in this clinical setting along with the potential diagnostic challenges.

Patient Findings: A 44-year-old man with a history of LT and hypothyroidism presented with an enlarging goiter. Initial imaging evaluation showed markedly enlarged gland with bilateral cervical and mediastinal adenopathy. Histopathologic evaluation confirmed the diagnosis of primary thyroid lymphoma on a background of LT. An ${ }^{18} \mathrm{~F}-\mathrm{FDG}$ PET/CT revealed increased uptake in the gland and lymph nodes. Follow-up ${ }^{18} \mathrm{~F}-$ FDG PET/CT after chemotherapy showed interval decrease in FDG uptake in the thyroid gland associated with interval decrease in the size and metabolic activity of the cervical and superior mediastinal lymph nodes.

Conclusions: The frequent association of LT with primary thyroid lymphoma and the overlap of their clinical and pathologic findings pose a significant diagnostic challenge. While other imaging techniques are helpful in evaluating anatomic local and regional extent of primary thyroid lymphoma, ${ }^{18}$ F-FDG PET/CT can be of an added value in evaluating its metabolic activity and detecting regional and distant disease as well as in assessing response to treatment.

\section{Introduction}

$\mathbf{P}$ RIMARY THYROID LYMPHOMA is an uncommon neoplasm frequently associated with lymphocytic thyroiditis (LT) (1-5). The overlap of their clinical and pathologic findings pose a significant diagnostic challenge. Once the pathologic diagnosis of primary thyroid lymphoma is established, imaging plays an important role in tumor staging and evaluating treatment response. We discuss the role of fluorodeoxyglucose positron emission tomography $\left({ }^{18} \mathrm{~F}\right.$-FDG PET)/CT in this clinical setting along with the potential diagnostic challenges.

\section{Patient}

A 44-year-old man with a history of LT and hypothyroidism presented with generalized urticaria, difficulty swallowing, and an enlarging goiter. Laboratory evaluation revealed an elevated C-reactive protein, positive antinuclear antibodies, a thyroglobulin antibody titer $>3000$ (normal
$<1: 100)$, a thyroid peroxidase antibody titer $>1000 \mathrm{U} / \mathrm{mL}$ (normal $<30 \mathrm{U} / \mathrm{mL}$ ), and a thyroid stimulating hormone level of $3.1 \mathrm{mIU} / \mathrm{L}$ (normal 0.30-5.50 mIU/L). Magnetic resonance imaging of the neck showed severe, diffuse thyromegaly with an overall gland size of $6 \times 12 \times 11 \mathrm{~cm}$ with a mass effect upon the upper esophagus and numerous prominent lymph nodes in the superior mediastinum (Fig. 1a). A subsequent contrastenhanced computed tomography (CT) examination of the neck and chest revealed multiple enlarged cervical and anterior mediastinal lymph nodes (Fig. 1b). A core needle biopsy of the thyroid revealed diffuse lymphocytic infiltration with scant thyroid follicles and a partial right thyroid lobectomy demonstrated markedly effaced architecture. The thyroid gland was replaced by a lymphoid infiltrate of small cells with irregular nuclear contours and clumped chromatin (Fig. 2). Occasional reactive germinal centers were present, consistent with residual LT. Thyroid epithelium was difficult to identify, but small foci were present and readily identified with thyroid transcription factor 1 (TTF1) immunostaining. Histopathologic

\footnotetext{
${ }^{1}$ Division of Nuclear Medicine, Department of Radiology; ${ }^{3}$ Department of Pathology; ${ }^{4}$ Division of Hematology/Oncology, Department of Internal Medicine; University of Michigan Health Systems, Ann Arbor, Michigan.

${ }^{2}$ Department of Nuclear Medicine, Department of Veterans Affairs Ann Arbor Medical Center, Ann Arbor, Michigan.
} 
FIG. 1. (a) Axial T2-weighted image of the neck at the level of the thyroid gland demonstrating massive heterogeneous enlargement of the gland extending posterior the larynx. An enlarged lymph node is noted in the left posterior cervical chain (arrows). (b) Coronal contrast enhanced computed tomography (CT) image showing a massively enlarged thyroid gland displacing the carotid arteries laterally. Note the left lower cervical enlarged lymph node (arrow).

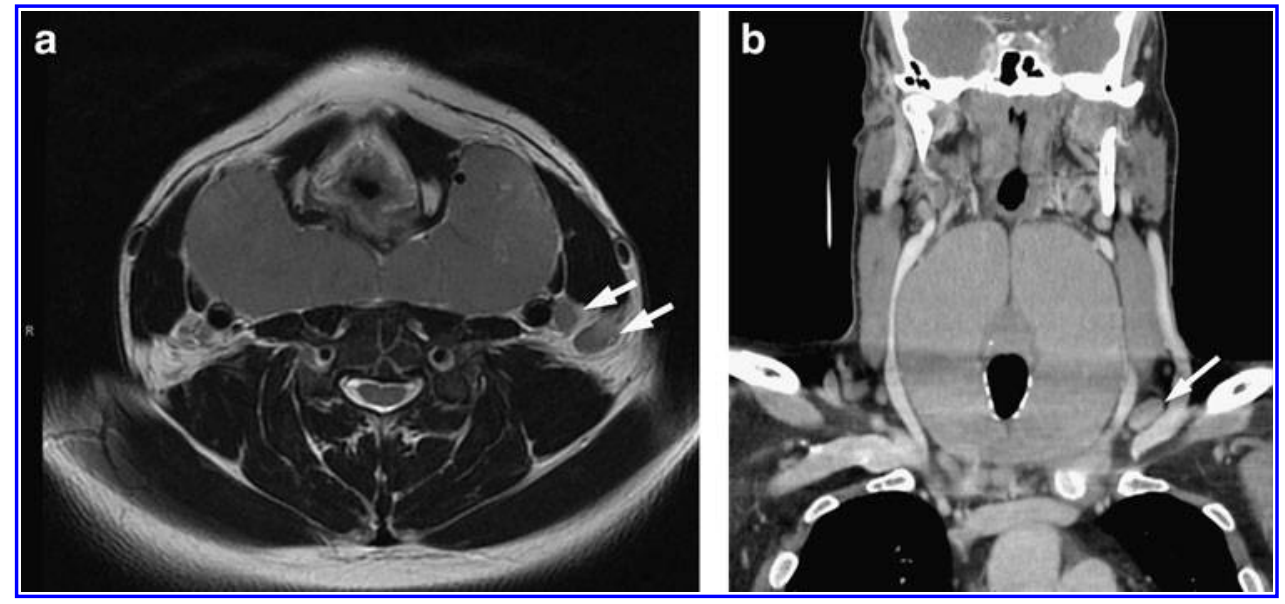

review of the bone marrow revealed a subtle, primarily interstitial infiltrate of atypical lymphocytes. Atypical circulating lymphocytes were present in the blood. Flow cytometry was performed on the thyroid tissue and bone marrow. The neoplastic T- lymphocytes expressed CD2, CD3, CD4, CD5, and CD7 (dim). Molecular studies performed on the thyroid tissue and bone marrow demonstrated a T-cell receptor gamma gene rearrangement. The differential diagnosis based on morphology and immunophenotype included adult T-cell leukemia/lymphoma (ATLL), Sezary syndrome, T-cell polymorphocytic leukemia (T-PLL), and a peripheral T-cell lymphoma. A negative study for human T-cell lymphotrophic virus 1 (HTLV-1) excluded ATLL. T-PLL typically presents with a higher white blood cell count and cytogenetic studies revealed no evidence of characteristic translocations. There were no skin lesions or erythroderma to suggest Sezary syndrome. Consequently, the features were most consistent with a peripheral T-cell lymphoma, in leukemic phase.

A ${ }^{18}$ F-FDG PET/CT scan was performed for further evaluation and staging. This showed a diffusely enlarged thyroid with heterogeneous increased FDG accumulation with max-

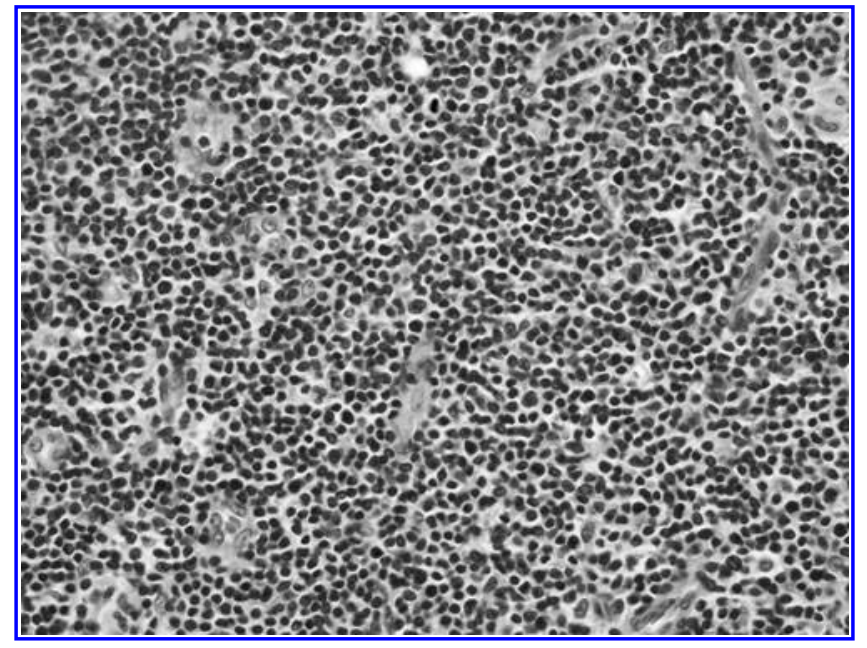

FIG. 2. Section of thyroid showing a marked infiltrate of monotonous small lymphocytes with irregular nuclear contours (hematoxylin and eosin, $400 \times$ magnification). imum standardized uptake $\left(\mathrm{SUV}_{\max }\right)$ of 9 compared to average mediastinal blood pool uptake $\left(\mathrm{SUV}_{\mathrm{avg}}\right)$ of 1.2 , with multiple bilateral FDG-avid cervical lymph nodes in levels II through $\mathrm{V}$ and in the superior mediastinum (Fig. 3). A repeat FDG PET/CT (Fig. 4) following three cycles of cyclophosphamide hydroxydaunorubicin oncovin prednisone (CHOP) chemotherapy revealed an interval decrease in FDG avidity involving the massively enlarged thyroid gland with $\mathrm{SUV}_{\max }$ of 6.6 (mediastinal blood pool SUV $\mathrm{Svg}_{\text {avg }}$ of 1.5), associated interval decrease in size, and metabolic activity of the cervical and superior mediastinal lymph nodes, suggesting a partial therapeutic response to chemotherapy.

\section{Discussion}

Occurring with a slight female predominance and a peak age incidence between 50 and 80 years, primary thyroid lymphoma is rare, representing $2 \%-5 \%$ of all thyroid malignancies and $<2 \%$ of extra-nodal lymphoma $(1,2)$. Thyroid lymphoma is most commonly associated with LT, with an overall 67-80-fold higher risk of developing thyroid lymphoma compared to the general population. Additionally, $0.5 \%$ of patients with LT will develop thyroid lymphoma (3$5)$. The time interval between diagnosis of LT and the development of thyroid lymphoma is $\sim 10$ years (4). The most common type of thyroid lymphoma is diffuse large B-cell lymphoma, which accounts for almost $70 \%$ of cases (6); however, various histopathologic subtypes have been reported in primary thyroid lymphoma, including low-grade lymphoma of mucosa-associated lymphoid tissue, Hodgkin's lymphoma, Burkitt's lymphoma, and peripheral T-cell lymphoma $(1,2)$. Patients present with a rapidly enlarging goiter, dysphonia, dysphagia, pain, and hoarseness with constitutional symptoms of malaise, fever, and weight loss $(1,2)$. Initial diagnosis is often suggested, but not confirmed by fineneedle aspiration, due to difficulty in distinguishing an infiltrating lymphoma from background LT. Flow cytometry can sometimes be useful in evaluating lymphoma in fine needle aspirates; however, larger tissue specimens obtained by core biopsy and/or surgical resection are usually required for definitive tissue diagnosis and cellular subclassification (2).

Although ultrasound imaging of the thyroid gland may play a role in the initial diagnosis of thyroid lymphoma, it has limited specificity given variable sonographic features 


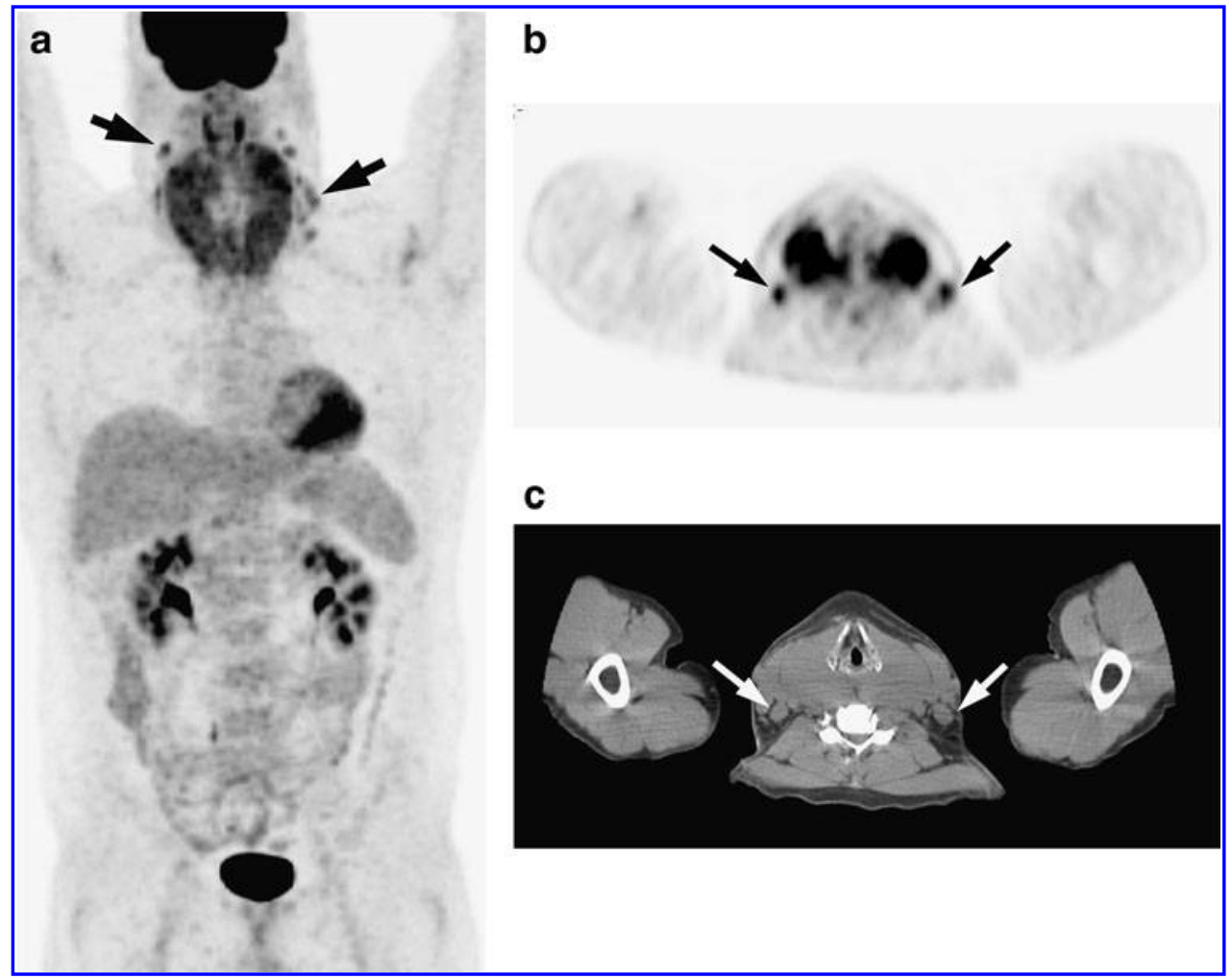

FIG. 3. Initial staging FDG PET/CT: (a) maximum intensity projection image, (b) axial PET, and (c) corresponding axial CT images demonstrating diffuse heterogeneous metabolic activity throughout the enlarged thyroid gland and bilateral hypermetabolic cervical and superior mediastinal lymph nodes (arrows).

and significant overlap with other benign and malignant conditions $(3,7)$.

FDG PET plays an important role in the initial staging and therapeutic response assessment of lymphoma in general due to its predictive prognostic value of long-term survival. Interim visual changes in uptake and differences in the stan- dardized uptake values between the initial and follow-up scans after chemotherapy have been shown to predict outcome (8). Recent reports have described the clinical utility of FDG PET in the evaluation of primary thyroid lymphoma (9-11). Basu et al. reported that FDG PET could be sensitive in evaluating disease activity at initial staging and follow-up in

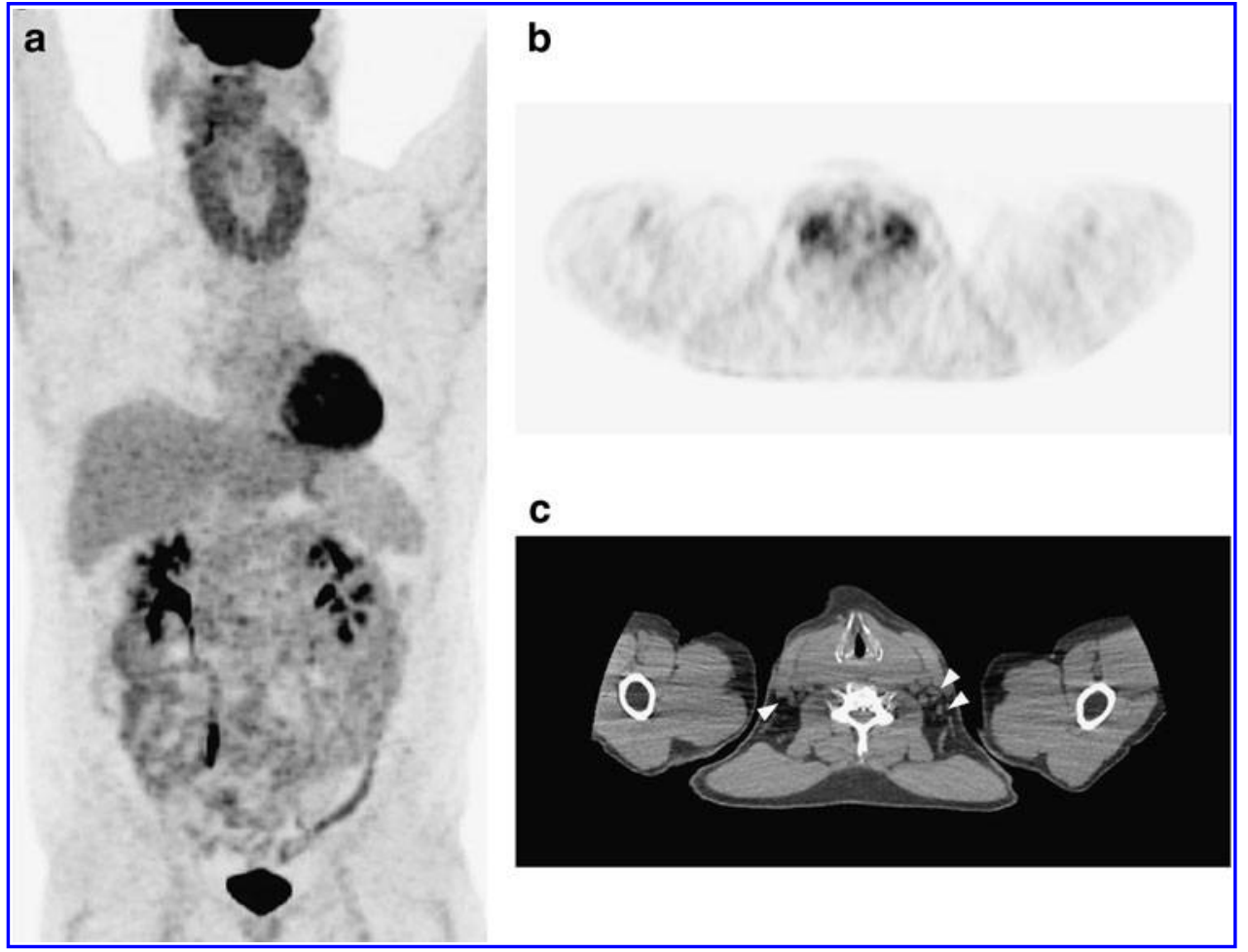

FIG. 4. Follow-up

fluorodeoxyglucose positron emission tomography (FDG PET)/CT following three cycles of chemotherapy: (a) maximum intensity projection image, (b) axial PET, and (c) corresponding axial CT images demonstrating an interval decrease in the FDG uptake in the thyroid gland and interval decrease in the size and metabolic activity of the cervical lymph nodes (arrowheads). 
six patients with suspected primary thyroid lymphoma, and suggested that FDG PET may detect disease recurrence earlier than CT alone. Lin also reported the value of FDG PET/CT in assessing responses to therapy in primary thyroid lymphoma (10). However, focal FDG accumulation is seen in thyroid adenomas and thyroid cancers of all types and diffuse FDG uptake in LT mimicking primary thyroid lymphoma limits the diagnostic specificity of FDG PET $(9,12,13)$. Massive thyroid enlargement associated with diffuse and markedly avid FDG uptake and the presence of cervical lymph nodes aided in the distinction of primary lymphoma from LT in the present case. However, the frequent association of LT with PL and the overlap of their clinical and pathologic findings pose a significant challenge, requiring both a high clinical suspicion and an extensive diagnostic approach in a patient with a rapidly enlarging thyroid and pre-existing LT. While other imaging techniques are helpful in evaluating anatomic local and regional extent of primary thyroid lymphoma, ${ }^{18}$ F-FDG PET/ $\mathrm{CT}$ can be of an added value in evaluating its metabolic activity and detecting regional and distant disease as well as in assessing response to treatment.

\section{Disclosure Statement}

The authors declare that no competing financial interests exist.

\section{References}

1. Ansell SM, Grant CS, Habermann TM 1999 Primary thyroid lymphoma. Semin Oncol 26:316-323.

2. Sarinah B, Hisham AN 2010 Primary lymphoma of the thyroid: diagnostic and therapeutic considerations. Asian I Surg 33:20-24.

3. Anderson L, Middleton WD, Teefey SA, Reading CC, Langer JE, Desser T, Szabunio MM, Mandel SJ, Hildebolt CF, Cronan JJ 2010 Hashimoto thyroiditis: part 2, sonographic analysis of benign and malignant nodules in patients with diffuse Нashimoto thyroiditis. AJR Am J Roentgenol 195:216-222.

4. Ben-Ezra J, Wu A, Sheibani K 1988 Hashimoto's thyroiditis lacks detectable clonal immunoglobulin and $\mathrm{T}$ cell receptor gene rearrangements. Hum Pathol 19:1444-1448.

5. Holm LE, Blomgren $\mathrm{H}$, Lowhagen $\mathrm{T} 1985$ Cancer risks in patients with chronic lymphocytic thyroiditis. $\mathrm{N}$ Engl J Med 312:601-604.
6. Widder S, Pasieka JL 2004 Primary thyroid lymphomas. Curr Treat Options Oncol 5:307-313.

7. Anderson L, Middleton WD, Teefey SA, Reading CC, Langer JE, Desser T, Szabunio MM, Hildebolt CF, Mandel SJ, Cronan JJ 2010 Hashimoto thyroiditis: part 1, sonographic analysis of the nodular form of Hashimoto thyroiditis. AJR Am J Roentgenol 195:208-215.

8. Casasnovas RO, Meignan M, Berriolo-Riedinger A, Bardet $S$, Julian A, Thieblemont C, Vera P, Bologna S, Brière J, Jais JP, Haioun C, Coiffier B, Morschhauser F 2011 SUVmax reduction improves early prognosis value of interim positron emission tomography scans in diffuse large B-cell lymphoma. Blood 118:37-43.

9. Basu S, Li G, Bural G, Alavi A 2009 Fluorodeoxyglucose positron emission tomography (FDG-PET) and PET/ computed tomography imaging characteristics of thyroid lymphoma and their potential clinical utility. Acta Radiol 50:201-204.

10. Lin EC 2007 FDG PET/CT for assessing therapy response in primary thyroid lymphoma. Clin Nucl Med 32:152153.

11. Marchesi M, Biffoni M, Biancari F 2004 False-positive finding on 18F-FDG PET after chemotherapy for primary diffuse large B-cell lymphoma of the thyroid: a case report. Ipn J Clin Oncol 34:280-281.

12. Cohen MS, Arslan N, Dehdashti F, Doherty GM, Lairmore TC, Brunt LM, Moley JF 2001 Risk of malignancy in thyroid incidentalomas identified by fluorodeoxyglucose-positron emission tomography. Surgery 130:941-946.

13. Cohen T, Krausz Y, Nissan A, Ben-Yehuda D, Klein M, Freund HR 2006 18F-fluorodeoxyglucose-avid thyroid incidentalomas in patients with lymphoma. Isr Med Assoc J 8:720-721.

Address correspondence to: Mohammad Arabi, M.D.

Division of Nuclear Medicine Department of Radiology University of Michigan Health System 1500 East Medical Center Drive, B1G G505 Ann Arbor, MI 48109-5028

E-mail: marabi@med.umich.edu 
This article has been cited by:

1. Massimo Bongiovanni, Luca Mazzucchelli, Vittoria Martin, Stefano Crippa, Martin Bolli, Sergio Suriano, Luca Giovanella. 2012. Images in Endocrine Pathology: a Starry-Sky in the Thyroid. Endocrine Pathology . [CrossRef] 